\title{
Baseline Characteristics and Outcomes Among Patients with Complicated Skin and Soft Tissue Infections Admitted to the Intensive Care Unit: Analysis of the Phase 3 COVERS Randomized Trial of Ceftaroline Fosamil Versus Vancomycin Plus Aztreonam
}

Miguel Sánchez-García · Jennifer Hammond · Jean Li Yan •

Michal Kantecki · Wajeeha Ansari · Matthew Dryden

Received: December 31, 2019/Published online: June 30, 2020

(c) Pfizer Inc. 2020

\section{ABSTRACT}

Aim: Exploratory analyses evaluated patient characteristics and outcomes among patients with complicated skin and soft tissue infection (cSSTI) in the phase 3 COVERS study who were admitted to an intensive care unit (ICU).

Methods: Adults with cSSTI (surface area $\geq 75$ $\mathrm{cm}^{2}$ ) and evidence of systemic inflammation and/or underlying comorbidities were randomized $2: 1$ to intravenous ceftaroline fosamil

Digital Features To view digital features for this article go to https://doi.org/10.6084/m9.figshare. 12006168.

Electronic supplementary material The online version of this article (https://doi.org/10.1007/s40121020-00297-3) contains supplementary material, which is available to authorized users.

M. Sánchez-García ( $\square)$

Hospital Clínico San Carlos, Calle del Prof Martín Lagos, Madrid, Spain

e-mail: miguelsanchez.hcsc@gmail.com

J. Hammond

Pfizer, Collegeville, PA, USA

J. L. Yan · W. Ansari

Pfizer, New York, NY, USA

M. Kantecki

Pfizer, Paris, France

M. Dryden

Royal Hampshire County Hospital, Winchester, UK
(600 mg every $8 \mathrm{~h} \quad$ [q8h]) or vancomycin $(15 \mathrm{mg} / \mathrm{kg}$ every $12 \mathrm{~h})$ plus aztreonam $(1 \mathrm{~g} \mathrm{q} 8 \mathrm{~h})$ for 5-14 days. Clinical response and ICU length of stay (LOS) within first hospitalization were evaluated in the modified intent-to-treat (MITT) and clinically evaluable (CE) populations; a Cox proportional hazards model identified factors associated with increased hospital LOS.

Results: Overall, 42 of 761 randomized patients were admitted to the ICU (ceftaroline fosamil, $n=32$; vancomycin plus aztreonam, $n=10$ ) prior to, or at start of, study treatment. Baseline differences between the ICU and non-ICU populations were indicative of more severe disease in ICU patients; within this subset, there were also some notable imbalances between treatment groups. Clinical cure rates at test-ofcure (ceftaroline fosamil vs. vancomycin plus aztreonam) were generally similar in the nonICU and ICU subsets (MITT population 79\% vs. $79 \%$ and $69 \%$ vs. $90.0 \%$, respectively; CE population $87 \%$ vs. $85 \%$ and $80 \%$ vs. $89 \%$, respectively). Median ICU LOS was 8 vs. 13 days, respectively. ICU admission was a risk factor predicting increased hospital LOS $(P<0.001)$.

Conclusions: Clinical outcomes for patients admitted to the ICU were generally similar to non-ICU patients, despite more severe baseline disease, with shorter median treatment duration in the ceftaroline fosamil group. ICU admission was associated with longer hospital LOS. Given the small sample size and 
unbalanced patient and disease characteristics within the ICU subgroup, differences between treatment groups should be interpreted with caution.

Trial registration: ClinicalTrials.gov identifier, NCT01499277.

Keywords: Antimicrobial resistance; Ceftaroline fosamil; Complicated skin and soft tissue infections; Healthcare resource use; Intensive care unit

\section{Key Summary Points}

\section{Why carry out this study?}

Ceftaroline fosamil is a $\beta$-lactam antibiotic with in vitro activity against methicillinresistant Staphylococcus aureus (MRSA). The previously reported phase 3 CANVAS 1 and 2 program supported approval of the standard ceftaroline fosamil dosage regimen (600 mg 1-h IV infusions q12h) in patients with complicated skin and soft tissue infection (cSSTI). The phase 3 COVERS study subsequently demonstrated the efficacy and safety of a high dose regimen of ceftaroline fosamil (600 mg 2-h IV infusions q8h) versus vancomycin plus aztreonam in hospitalized patients with cSSTI and underlying co-morbidities.

Exploratory analyses of the COVERS study, reported here, evaluated the length of stay in hospital and any time spent in the intensive care unit (ICU); a hypothesis was not pre-specified.

\section{What was learned from the study?}

In the overall COVERS study, high-dose ceftaroline fosamil was shown to be noninferior to vancomycin plus aztreonam in the primary efficacy analyses, with comparable clinical cure rates at the testof-cure (TOC) visit between treatment groups. Patients admitted to the ICU had signs of more severe disease than non-ICU patients, with some imbalances between treatment groups. Clinical outcomes at TOC in the ICU subset were broadly consistent with those in non-ICU patients, with wider confidence intervals for between-group differences reflecting the small numbers of patients in the ICU subset. There was no difference between treatment groups in the median hospital length of stay (LOS); however, ICU admission was an independent predictor for increased hospital LOS.

Although patients admitted to the ICU in COVERS had more severe disease, clinical outcomes for ICU patients were generally similar to those in non-ICU patients. Within the ICU subset, median hospital and ICU LOS were shorter for ceftaroline fosamil (8 days) compared with vancomycin plus aztreonam (13 days) overall, and median ICU LOS was shorter for ceftaroline fosamil across all primary cSSTI diagnoses.

\section{INTRODUCTION}

Complicated skin and soft tissue infection (cSSTI), also referred to as acute bacterial skin and skin structure infection (ABSSSI), which includes a broad range of acute and chronic skin conditions, such as secondary infections of diseased skin, surgical/other wound infections, cellulitis with/without abscess, necrotizing fasciitis, and myonecrosis, are collectively among the most common bacterial infections 
observed in hospitalized patients, and can be associated with significant morbidity and mortality [1-3] as well as patient admission to the intensive care unit (ICU) [4-6]. In a retrospective observational study (REACH), mean length of hospital stay (37.1 vs. 15.8 days), time to clinical stability (16.8 vs. 9.3 days), and total mortality rate $(16.9 \%$ vs. $2.5 \%)$ were higher in patients with cSSTI admitted to the ICU than in those who were not, indicating the vulnerability of this patient subset [7].

Typical treatment for cSSTI involves surgical intervention and broad-spectrum intravenous (IV) antimicrobials [1, 3, 8]. Early surgical source control is a main determinant of outcome, particularly in more severe cases of cSSTI, and prompt and appropriate empiric antibiotic treatment contributes to reducing the morbidity and mortality associated with cSSTI $[1,8]$. Gram-positive pathogens, including Staphylococcus aureus and group A streptococci, are frequent causes of cSSTI, but Gram-negative pathogens, such as Escherichia coli and Pseudomonas aeruginosa, as well as enterococci and anaerobic bacteria, may also be implicated [2]. Adequacy of empiric antimicrobial therapy is challenged in many parts of the world by the prevalence of resistant Gram-positive pathogens, such as methicillin-resistant S. aureus (MRSA), which limits the available treatment options [9].

Ceftaroline, the active form of the prodrug ceftaroline fosamil, is a cephalosporin with broad in vitro activity against Gram-positive bacteria, including MRSA, and common Gramnegative bacteria that do not express extendedspectrum $\beta$-lactamases (ESBLs). It is approved in Europe, the United States, and various other countries for the treatment of adults and children aged $>2$ years with cSSTI or communityacquired pneumonia (excluding pneumonia caused by MRSA) [10]. The initial adult phase 3 cSSTI trials evaluated ceftaroline fosamil $600 \mathrm{mg}$ every $12 \mathrm{~h}$ (q12h) versus vancomycin plus aztreonam [11, 12]. A subsequent multicenter randomized phase 3 trial (COVERS; NCT01499277) compared the efficacy and safety of ceftaroline fosamil $600 \mathrm{mg}$ every $8 \mathrm{~h}$ (q8h) with vancomycin plus aztreonam for the treatment of hospitalized adult patients with
cSSTI and extensive cutaneous involvement, including evidence of systemic inflammation or underlying comorbidities associated with impaired immune response [13]. A small subset of patients in COVERS with severe disease were admitted to the ICU. Comparative data describing the clinical and disease characteristics of patients who were and were not admitted to the ICU in COVERS are presented here. In addition, exploratory analyses describing healthcare resource-related outcomes, including length of hospitalization and length of ICU stay, are discussed.

\section{METHODS}

\section{Study Design}

The main methods and results of the COVERS study have been reported previously [13]. In brief, patients aged $\geq 18$ years with cSSTI and evidence of systemic inflammation and/or underlying comorbidities were randomized 2:1 to receive IV ceftaroline fosamil $600 \mathrm{mg}$ (2-h IV infusions) q8h or vancomycin $15 \mathrm{mg} / \mathrm{kg}$ (2-h IV infusions) $\mathrm{q} 12 \mathrm{~h}$ plus aztreonam $1 \mathrm{~g}$ (30-min IV infusions) q8h for 5-14 days (for an overview of the study design, see Fig. S1). Patients with moderate renal impairment [creatinine clearance $(\mathrm{CrCL}) \geq 20$ to $<50 \mathrm{~mL} / \mathrm{min}$; estimated by the Cockcroft-Gault formula [14]] received an adjusted dose of study drug as determined by an unblinded pharmacist/staff member; patients with estimated $\mathrm{CrCL}<20 \mathrm{~mL} / \mathrm{min}$ were excluded from the study. See Text $\mathrm{S} 2$ for a description of baseline assessments. The protocol was approved by each study site's independent ethics committees/institutional review board, and the study was conducted in accordance with the Declaration of Helsinki and the International Conference on Harmonization/Good Clinical Practice. All patients provided written informed consent.

\section{Patients}

cSSTI was defined as extensive cellulitis, major cutaneous abscess (limited to $30 \%$ of the trial 
population per the US Food and Drug Administration guidelines [15]), burn infection, or traumatic/surgical wound infection with purulent draining, with a minimum surface area of $75 \mathrm{~cm}^{2}$. For study inclusion, infection was to be of sufficient severity to warrant hospitalization and $\geq 5$ days of parenteral antibacterial therapy. Patients were also required to have one or more signs of systemic inflammatory response during the $24 \mathrm{~h}$ prior to first dose of the study drug (temperature $\geq 38.0{ }^{\circ} \mathrm{C}$ or $\leq 36.0{ }^{\circ} \mathrm{C}$; white blood cells $>12,000$ cells $/ \mathrm{mm}^{3},<4000$ cells/ $\mathrm{mm}^{3}$ or $>10 \%$ band forms; heart rate $>90$ beats per min and respiratory rate $>20$ breaths per min after 10 min of rest) and/or one or more of the following underlying comorbidities associated with impaired immune response: diabetes mellitus requiring treatment with drugs; stage 2 or 3 human immunodeficiency virus (HIV) infection; chronic renal impairment (estimated $\mathrm{CrCL} \geq 20$ to $<50 \mathrm{~mL} / \mathrm{min}$ ); cirrhosis; peripheral vascular disease; malnutrition; use of immunosuppressive agents; and malignancy (with the exception of non-melanoma skin cancers). Patients with systemic inflammatory response syndrome (SIRS), and those with severe sepsis were also eligible for inclusion. Patients were excluded from the study if they had signs of septic shock or if their body weight exceeded $130 \mathrm{~kg}$. Concomitant systemic antimicrobial agents with potential efficacy in cSSTI were not permitted; patients who received systemic antimicrobials for $>24 \mathrm{~h}$ within the 96 $\mathrm{h}$ prior to first study drug administration were excluded from the study. ICU admission was based on investigators' judgement of each patient's clinical condition as well as any relevant institutional guidelines or protocols; there were no study-specific criteria for ICU admission or targets for enrollment of ICU patients, and randomization was not stratified by treatment location.

\section{Outcome Measures}

Clinical outcomes (see Table S3 for clinical response definitions) were assessed at end of treatment (EOT) and at a test-of-cure (TOC) visit (8-15 days after last dose of study drug) in the modified intent-to-treat (MITT) and clinically evaluable (CE) populations (see Table S4 for details of analysis populations). The betweengroup difference in clinical cure rates at the TOC visit was the primary efficacy outcome [13]. Safety assessments included the incidence and intensity of adverse events (AEs), as well as physical examinations, vital signs, and clinically important changes in chemistry, hematology, and urinalysis laboratory values up to the late follow-up (LFU) visit (21-35 days after the final dose of study drug) [13]. The location/ ward of each patient was recorded daily. Healthcare resource utilization variables, including hospital length of stay (LOS) and ICU LOS during the first hospitalization, were assessed as exploratory objectives.

\section{Statistical Methods}

The main statistical methods, including noninferiority assessments for the primary efficacy evaluation of clinical cure at the TOC visit, have been reported [13]. Patient demographics and baseline characteristics data, and efficacy and safety results for the ICU and non-ICU subsets, were summarized descriptively. For the exploratory analyses, an unadjusted Kaplan-Meier survival function was used to measure the mean hospital and ICU LOS during the first admission, and LOS was compared between treatment groups using a Wilcoxon test. A Cox proportional hazards model was used to identify factors associated with increased hospital LOS using a generalized score test of chi-square test statistic.

\section{RESULTS}

\section{Patients}

Overall, 802 patients were enrolled between May 2012 and June 2014, and 761 were randomized to receive ceftaroline fosamil $(n=506)$ or vancomycin plus aztreonam $(n=255)$ [13]. Of the 761 randomized patients, 42 were admitted to the ICU prior to, or at the start of, study drug administration (ceftaroline fosamil, 
Table 1 Baseline patient demographic and disease characteristics in ICU and non-ICU patients (MITT population)

\begin{tabular}{|c|c|c|c|c|}
\hline \multirow[t]{2}{*}{ Parameter } & \multicolumn{2}{|c|}{ ICU patients, $n(\%)$} & \multicolumn{2}{|c|}{ Non-ICU patients, $n(\%)$} \\
\hline & $\begin{array}{l}\text { Ceftaroline } \\
\text { fosamil } \\
(n=32)\end{array}$ & $\begin{array}{l}\text { Vancomycin }+ \\
\text { aztreonam } \\
(n=10)\end{array}$ & $\begin{array}{l}\text { Ceftaroline } \\
\text { fosamil } \\
(n=474)\end{array}$ & $\begin{array}{l}\text { Vancomycin }+ \\
\text { aztreonam } \\
(n=245)\end{array}$ \\
\hline Mean (SD) age, years & $54.4(17.1)$ & $64.1(13.0)$ & $52.4(16.5)$ & $53.2(16.2)$ \\
\hline \multicolumn{5}{|l|}{ Sex, $n(\%)$} \\
\hline Male & $18(56.3)$ & $6(60.0)$ & $292(61.6)$ & $142(58.0)$ \\
\hline \multicolumn{5}{|l|}{ Primary cSSTI diagnosis, $n(\%)$} \\
\hline Cellulitis & $8(25.0)$ & $2(20.0)$ & $292(61.6)$ & $134(54.7)$ \\
\hline Traumatic or surgical wound infection & $4(12.5)$ & $0(0.0)$ & $59(12.4)$ & $41(16.7)$ \\
\hline Major cutaneous abscess & $0(0.0)$ & $0(0.0)$ & $103(21.7)$ & $59(24.1)$ \\
\hline Burn infection & $20(62.5)$ & $8(80.0)$ & $18(3.8)$ & $10(4.1)$ \\
\hline Other & $0(0.0)$ & $0(0.0)$ & $2(0.4)$ & $1(0.4)$ \\
\hline Median lesion size, $\mathrm{cm}^{2}$ & 702.0 & 1327.0 & 379.5 & 375.0 \\
\hline SIRS, $n(\%)$ & $8(25.0)$ & $3(30.0)$ & $191(40.3)$ & $102(41.6)$ \\
\hline Presence of bacteremia, $n(\%)$ & $3(9.4)$ & $3(30.0)$ & $15(3.2)$ & $13(5.3)$ \\
\hline \multicolumn{5}{|l|}{ Comorbid conditions, $n(\%)$} \\
\hline Diabetes mellitus & $2(6.3)$ & $2(20.0)$ & $82(17.3)$ & $36(14.7)$ \\
\hline Peripheral vascular disease & $2(6.3)$ & $0(0.0)$ & $25(5.3)$ & $11(4.5)$ \\
\hline Diabetes mellitus and peripheral vascular disease & $0(0.0)$ & $0(0.0)$ & $7(1.5)$ & $3(1.2)$ \\
\hline HIV infection & $0(0.0)$ & $0(0.0)$ & $6(1.3)$ & $2(0.8)$ \\
\hline Renal impairment & $1(3.1)$ & $0(0.0)$ & $14(3.0)$ & $5(2.0)$ \\
\hline Cirrhosis & $0(0.0)$ & $0(0.0)$ & $1(0.2)$ & $2(0.8)$ \\
\hline Malnutrition $^{\mathrm{a}}$ & $1(3.1)$ & $0(0.0)$ & $4(0.8)$ & $5(2.0)$ \\
\hline Use of immunosuppressive agents ${ }^{\mathrm{b}}$ & $0(0.0)$ & $0(0.0)$ & $6(1.3)$ & $4(1.6)$ \\
\hline $\begin{array}{l}\text { Malignancy other than non-melanoma skin } \\
\text { cancers }\end{array}$ & $2(6.3)$ & $0(0.0)$ & $10(2.1)$ & $5(2.0)$ \\
\hline Elevated WBC $\left(>12,000\right.$ cells $\left./ \mathrm{mm}^{3}\right), n(\%)^{\mathrm{c}}$ & $10(31.3)$ & $3(30.0)$ & $145(30.6)$ & $68(27.8)$ \\
\hline \multicolumn{5}{|l|}{ Platelet levels, $\times 10^{9} / \mathrm{L}^{\mathrm{d}}$} \\
\hline Mean (SD) & $269.1(109.0)$ & $264.0(87.7)$ & $261.4(94.9)$ & $258.7(115.5)$ \\
\hline Median (range) & $\begin{array}{l}266.0(140 \\
727)\end{array}$ & $231.5(177,395)$ & $\begin{array}{c}248.5(49 \\
600)\end{array}$ & $240.0(9,834)$ \\
\hline \multicolumn{5}{|l|}{ Creatinine levels, $\mathrm{mg} / \mathrm{dL}^{\mathrm{e}}$} \\
\hline Mean (SD) & $79.1(27.0)$ & $83.5(33.2)$ & $80.9(36.6)$ & $77.4(26.4)$ \\
\hline Median (range) & $75.0(43,150)$ & $75.5(47,131)$ & $74.0(31,376)$ & $73.0(26,284)$ \\
\hline
\end{tabular}


Table 1 continued

\begin{tabular}{|c|c|c|c|c|}
\hline \multirow[t]{2}{*}{ Parameter } & \multicolumn{2}{|c|}{ ICU patients, $n(\%)$} & \multicolumn{2}{|c|}{ Non-ICU patients, $n(\%)$} \\
\hline & $\begin{array}{l}\text { Ceftaroline } \\
\text { fosamil } \\
(n=32)\end{array}$ & $\begin{array}{l}\text { Vancomycin }+ \\
\text { aztreonam } \\
(n=10)\end{array}$ & $\begin{array}{l}\text { Ceftaroline } \\
\text { fosamil } \\
(n=474)\end{array}$ & $\begin{array}{l}\text { Vancomycin }+ \\
\text { aztreonam } \\
(n=245)\end{array}$ \\
\hline \multicolumn{5}{|l|}{ Bilirubin levels, $\mathrm{mg} / \mathrm{dL}^{\mathrm{f}}$} \\
\hline Mean (SD) & $11.7(8.4)$ & $10.3(5.2)$ & $10.5(7.4)$ & $10.7(8.9)$ \\
\hline Median (range) & $10.0(3,39)$ & $8.5(4,22)$ & $8.0(3,55)$ & $8.0(3,68)$ \\
\hline Respiratory rate $>20 \mathrm{bpm}, n(\%)^{\mathrm{g}}$ & $6(18.8)$ & $1(10.0)$ & $113(23.8)$ & $56(22.9)$ \\
\hline \multicolumn{5}{|l|}{$\mathrm{CrCL}(\mathrm{mL} / \mathrm{min}), n(\%)^{\mathrm{h}}$} \\
\hline$\geq 20$ to $\leq 30$ & 0 & 0 & $8(1.7)$ & $1(0.4)$ \\
\hline$>30$ to $\leq 50$ & $4(12.5)$ & $1(10.0)$ & $27(5.7)$ & $16(6.5)$ \\
\hline$>50$ & $27(84.4)$ & $9(90.0)$ & $426(89.9)$ & $220(89.8)$ \\
\hline Surgical intervention, $n$ (\%) & $1(3.1)$ & $0(0.0)$ & $11(2.3)$ & $5(2.0)$ \\
\hline Prior systemic antibiotics within 4 weeks, $n$ (\%) & $13(40.6)$ & $2(20.0)$ & $227(47.9)$ & $114(46.5)$ \\
\hline
\end{tabular}

bpm beats per minute, $B M I$ body mass index, $C r C L$ creatinine clearance, $c S S T I$ complicated skin and soft tissue infection, $H I V$ human immunodeficiency virus, ICU intensive care unit, MITT modified intent-to-treat, SD standard deviation, SIRS systemic inflammatory response syndrome, $W B C$ white blood cell

${ }^{a}$ Defined as baseline albumin $<0.25 \mathrm{~g} / \mathrm{L}$ in the absence of liver disease or baseline $\mathrm{BMI}<17 \mathrm{~kg} / \mathrm{m}^{2}$

b Immunosuppressive agents taken for $\geq 7$ days continuously prior to first dose

c Data missing for two patients in the ceftaroline fosamil ICU group, 52 patients in the ceftaroline fosamil non-ICU group, and 30 patients in the vancomycin plus aztreonam non-ICU group

${ }^{d}$ Data missing for two patients in the ceftaroline fosamil ICU group, two patients in the ICU vancomycin plus aztreonam group, 82 patients in the ceftaroline fosamil non-ICU group, and 48 patients in the vancomycin plus aztreonam non-ICU group

e Data missing for 1 patient in the ceftaroline fosamil ICU group, 13 patients in the ceftaroline fosamil non-ICU group, and 7 patients in the vancomycin plus aztreonam non-ICU group

${ }^{\mathrm{f}}$ Data missing for 3 patients in the ceftaroline fosamil ICU group, 54 patients in the ceftaroline fosamil non-ICU group, and 28 patients in the non-ICU vancomycin plus ceftaroline fosamil group

${ }^{g}$ Data missing for 7 patients in the ceftaroline fosamil non-ICU group

h Data missing for 1 patient in the ceftaroline fosamil ICU group, 13 patients in the ceftaroline fosamil non-ICU group, and 8 patients in the vancomycin plus aztreonam non-ICU group

$n=32$; vancomycin plus aztreonam, $n=10$ ); reasons for all ICU admissions were related to the disease under study. Baseline and disease characteristics for ICU and non-ICU patients, including primary cSSTI diagnosis and comorbidities, are summarized in Table 1.

The mean (SD) ages of patients in the ICU and non-ICU subsets (MITT population) were
57 (16.6) and 53 (16.4) years, respectively. The majority of patients in both groups were $<65$ years of age $[64.3 \% \quad(27 / 42)$ and $75.5 \%(543 / 719)$, respectively] and male [57.1\% (24/42) and 60.4\% (434/719), respectively]. Among the 42 patients admitted to the ICU, the primary cSSTI diagnosis was burn infection in $66.7 \%(28 / 42)$ and cellulitis in $23.8 \%$ (10/42). In 
contrast, the most common primary cSSTI diagnoses in the non-ICU subset were cellulitis [59.2\% (426/719)] and major cutaneous abscess [22.5\% (162/719)]. Median baseline lesion size was $899 \mathrm{~cm}^{2}$ for the ICU subset and $376 \mathrm{~cm}^{2}$ for the non-ICU subset. Bacteremia was present in $14.3 \%(6 / 42)$ of patients in the ICU subset and $3.9 \%(28 / 719)$ of patients in the non-ICU subset, and SIRS was present in $26.2 \%(11 / 42)$ and $40.8 \%(293 / 719)$ of patients, respectively. Systemic antibiotics within the 4 weeks before starting the study were received by $35.7 \%(15 /$ $42)$ and $47.4 \%(341 / 719)$ of patients in the ICU and non-ICU subsets, respectively.

Within the ICU subset, there were notable imbalances between the ceftaroline fosamil and vancomycin plus aztreonam treatment groups in some baseline and disease characteristics (Table 1). For example, burn infection was the predominant primary cSSTI diagnosis in both groups, but was more frequent in the vancomycin plus aztreonam group; bacteremia was also documented more frequently in the vancomycin plus aztreonam ICU group. In contrast, more than twice the proportion of patients in the ceftaroline fosamil ICU group (41\%) had received prior antibiotics compared with those in the vancomycin plus aztreonam ICU group (20\%).

\section{Baseline Pathogens}

Pathogens isolated at baseline from ICU and non-ICU patients are shown in Table S5. Pathogens most frequently isolated in ICU patients (MITT population) included S. aureus (25 patients [59.5\%], including 4 with methicillin-susceptible $S$. aureus and 21 with MRSA), streptococci [5 patients (11.9\%)], and Gramnegative Enterobacteriaceae [7 patients (16.7\%)]. No ESBL-positive organisms were identified in ICU patients. A similar baseline pathogen profile was observed in non-ICU patients, with $S$. aureus being the most commonly isolated (see Table S5); however, as would be expected for this much larger group, there was a wider and more diverse collection of pathogens in non-ICU patients, including some ESBL-positive Enterobacteriaceae isolates.
Minimum inhibitory concentration (MIC) distributions for ceftaroline and vancomycin against MSSA and MRSA isolates from ICU and non-ICU patients available for susceptibility testing (see Figure S6) showed that all isolates were within current respective European Committee on Antimicrobial Susceptibility Testing and Clinical and Laboratory Standards Institute MIC susceptibility breakpoints $[16,17]$.

\section{Clinical Outcomes}

In the ICU subset, the median (range) durations of exposure to ceftaroline fosamil and vancomycin plus aztreonam were 7 (3-14) and 10 (5-14) days, respectively, in the MITT population, and 8 (3-14) and 10 (5-14) days, respectively, in the CE population. Corresponding values for the non-ICU subset were $8(0-14)$ and 8 (0-14) days (MITT population), and 8 (2-14) and 8 (0-14) days (CE population). Clinical responses at the TOC visit (Table 2) were comparable between treatment groups in non-ICU patients (both 79\%). In the ceftaroline fosamil group, the clinical cure rate in the ICU subset (69\%) was lower than for non-ICU patients. In contrast, for vancomycin plus aztreonam the clinical cure rate was higher in the ICU subset (90\%) relative to non-ICU patients. Compared with non-ICU patients, the substantially wider 95\% confidence intervals for the estimates of differences in clinical cure rate between treatment groups in the ICU subset in both the MITT and CE populations (Table 2), reflect the much smaller sample size of the ICU subset.

\section{Safety Results}

An overview of AEs and serious AEs (SAEs) in ICU and non-ICU patients up to the LFU visit is shown in Table 3. In the ICU subset, there were six SAEs in six patients (18.3\%) in the ceftaroline fosamil group (one case each of myocardial infarction, pneumonia, abdominal infection, toxic epidermal necrolysis, peripheral arterial occlusive disease, and venous thrombosis), and one SAE in one patient $(10.0 \%)$ in the vancomycin plus aztreonam group (one case of pulmonary embolism). Three of the SAEs in the 


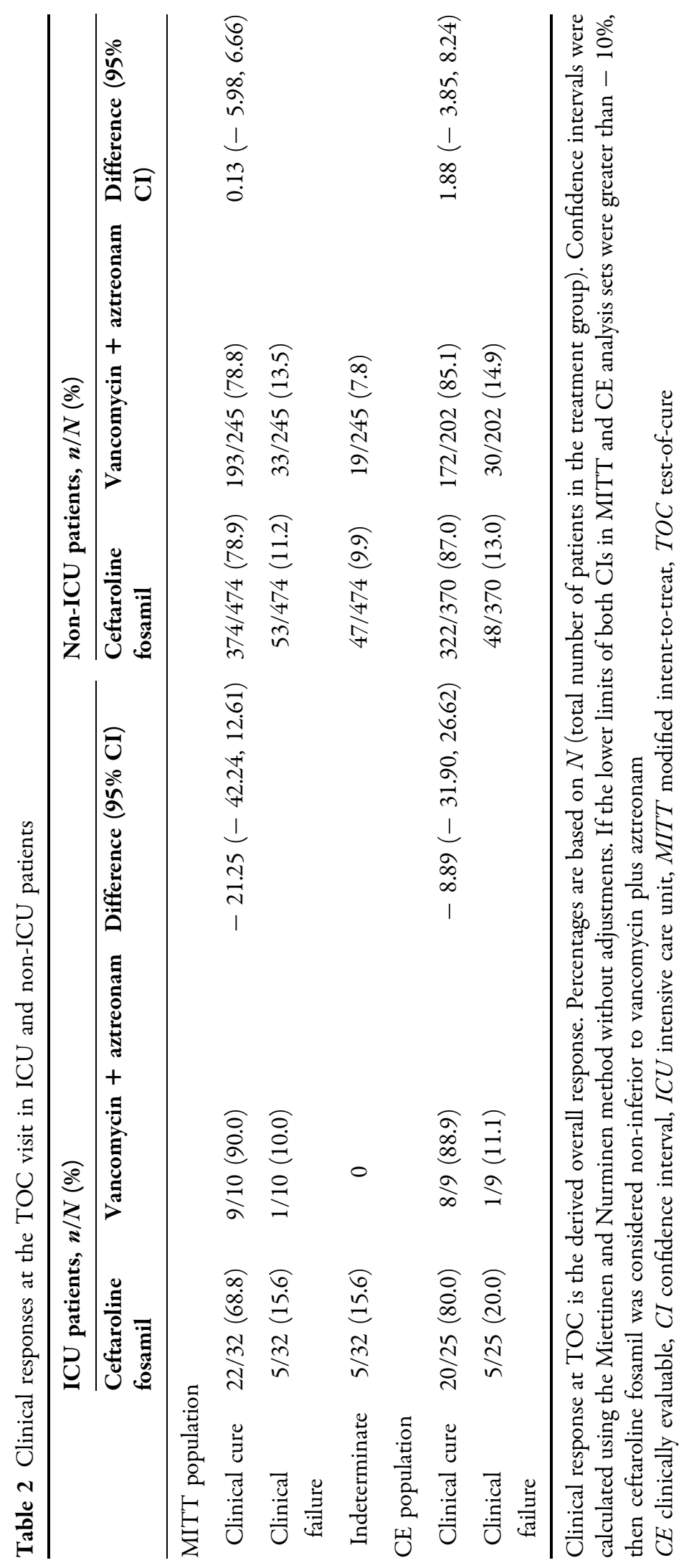


Table 3 Overview of adverse events in ICU and non-ICU patients (safety population)

\begin{tabular}{|c|c|c|c|c|}
\hline \multirow[t]{2}{*}{ Outcome } & \multicolumn{2}{|c|}{ ICU patients, $n(\%)$} & \multicolumn{2}{|c|}{ Non-ICU patients, $n(\%)$} \\
\hline & $\begin{array}{l}\text { Ceftaroline } \\
\text { fosamil } \\
(n=32)\end{array}$ & $\begin{array}{l}\text { Vancomycin }+ \\
\text { aztreonam } \\
(n=10)\end{array}$ & $\begin{array}{l}\text { Ceftaroline } \\
\text { fosamil } \\
(n=474)\end{array}$ & $\begin{array}{l}\text { Vancomycin }+ \\
\text { aztreonam } \\
(n=245)\end{array}$ \\
\hline Any AE & $16(50.0)$ & $5(50.0)$ & $216(45.6)$ & $111(45.3)$ \\
\hline $\begin{array}{l}\text { Any } \mathrm{AE} \text { causally related to study drug } \\
\text { by investigator }\end{array}$ & $2(6.3)$ & $1(10.0)$ & $79(16.7)$ & $41(16.7)$ \\
\hline Any $\mathrm{AE}$ with outcome of death & $1(3.1)$ & 0 & $2(0.4)$ & $2(0.8)$ \\
\hline $\begin{array}{l}\text { Any SAE (including events with } \\
\text { outcome of death) }\end{array}$ & $6(18.8)$ & $1(10.0)$ & $20(4.2)$ & $12(4.9)$ \\
\hline $\begin{array}{l}\text { Any AE leading to permanent } \\
\text { discontinuation of study treatment }\end{array}$ & $3(9.4)$ & 0 & $29(6.1)$ & $11(4.5)$ \\
\hline Any $\mathrm{AE}$ of severe intensity & $3(9.4)$ & 0 & $14(3.0)$ & $9(3.7)$ \\
\hline
\end{tabular}

Patients with multiple AEs in the same category are counted only once in that category. Patients with AEs in more than one category are counted once in each category

$A E$ adverse event, $I C U$ intensive care unit, $S A E$ serious adverse event

ceftaroline fosamil ICU subset (myocardial infarction, pneumonia and abdominal infection) were reported to be of severe intensity. None of the reported SAEs in either treatment group in the ICU subset were considered by the investigator to be related to study treatment.

\section{Exploratory Healthcare Resource Use Analyses}

In the overall COVERS study population, median (range) hospital LOS for both treatment groups in the MITT and CE populations was 11 days (MITT population: ceftaroline fosamil, 2-55 days; vancomycin plus aztreonam, 1-45 days; CE population: ceftaroline fosamil, 4-55 days; vancomycin plus aztreonam, 4-45 days) and there was no significant difference ( $P=0.802$ and $P=0.858$, respectively) between treatment groups (Fig. 1a). The relationships between exploratory variables and hospital LOS (assessed by Cox proportional hazards model) are summarized in Table 4 . Of note, ICU admission $(P<0.001)$ was a risk factor predicting increased hospital LOS in both the MITT and CE populations, as was the presence of comorbidities $(P<0.001$ and $P=0.003$, respectively).

Within the ICU subset, median hospital and ICU LOS were shorter for ceftaroline fosamil (8 days) compared with vancomycin plus aztreonam (13 days) overall, and median ICU LOS was shorter for ceftaroline fosamil across all primary cSSTI diagnoses (see Fig. 1b, Table S7).

\section{DISCUSSION}

This analysis summarizes the baseline characteristics and treatment outcomes by ICU admission status of patients enrolled in the phase 3 COVERS trial who were randomized to receive ceftaroline fosamil or vancomycin plus aztreonam. As might be expected, several differences between ICU and non-ICU patients were observed, reflecting greater disease severity in patients admitted to the ICU. Most notably, patients in the ICU subset presented with a greater median lesion size at baseline than nonICU patients, which has been identified as a risk factor for more severe disease [18]. Larger, rapidly progressing SSTI lesions typically require urgent management, monitoring and treatment 
A
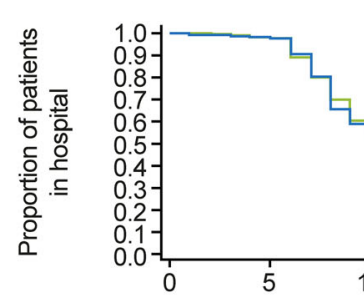

MITT population

Ceftaroline fosamil

Vancomycin plus aztreonam

Number of patients at risk Ceftaroline fosamil: 506

Vancomycin

plus aztreonam:

50

492

$300 \quad 170 \quad 63$

33

ngth of hospital stay, days
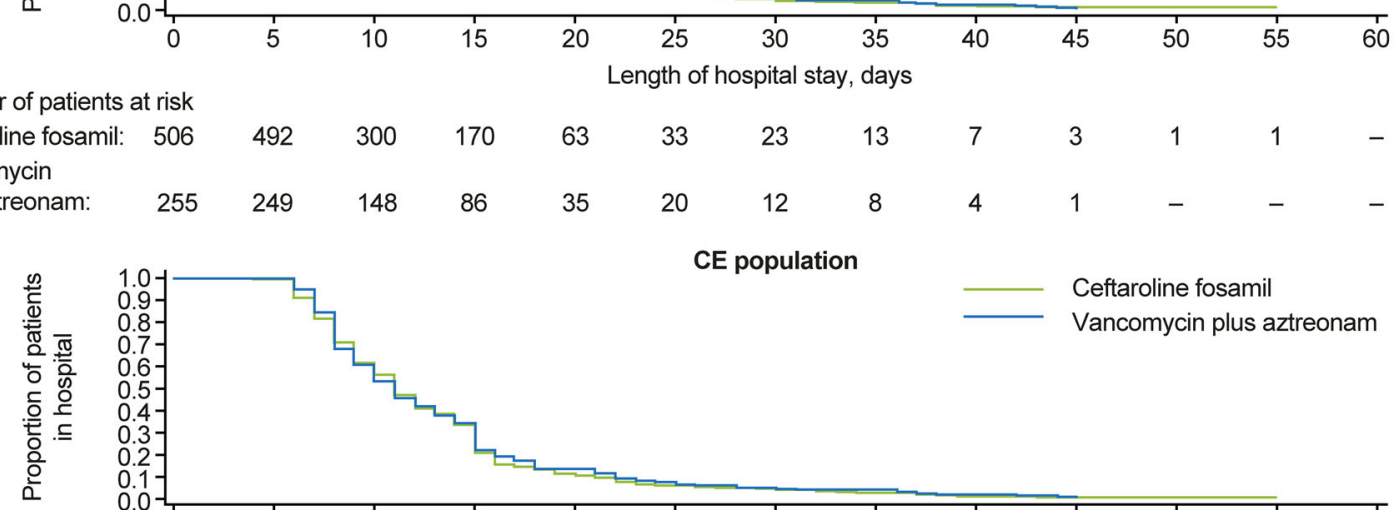

\title{
CE population
}

Ceftaroline fosamil

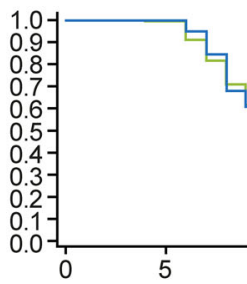

Number of patients at risk

Ceftaroline fosamil: 395

Vancomycin

plus aztreonam

B

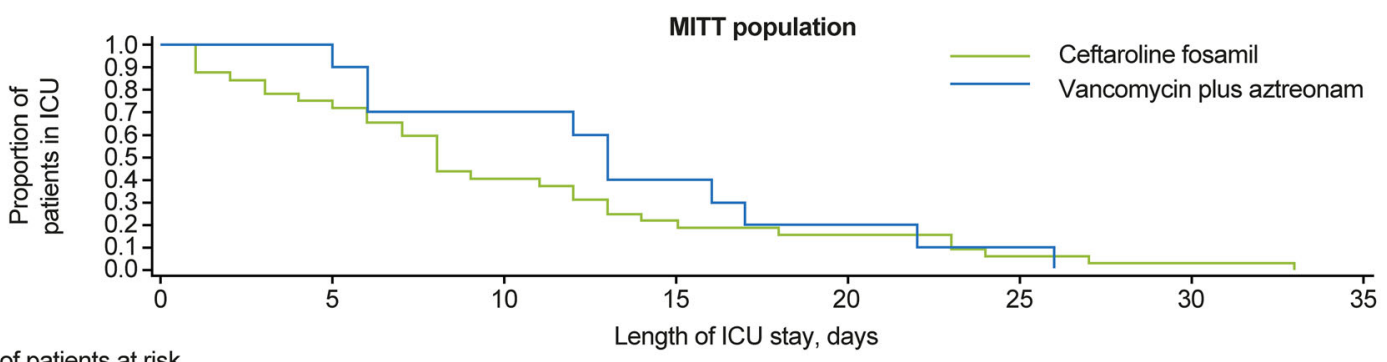

Number of patients at risk

Ceftaroline fosamil: 506

Vancomycin

plus aztreonam: 254

$\begin{array}{ll}24 & 13 \\ 254 & 7\end{array}$

Length of hospital stay, days

Vancomycin plus aztreonam
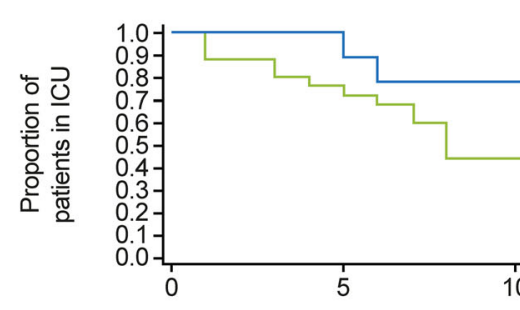

7

7
4

\begin{abstract}
5
\end{abstract}
2

CE population

Number of patients at risk

Ceftaroline fosamil: 395

Vancomycin

plus aztreonam: 210

$\begin{array}{cc}19 & 11 \\ 210 & 7\end{array}$

Length

gth of ICU stay, days 
4Fig. 1 Kaplan-Meier curves for a length of first hospital stay; b length of ICU stay. ${ }^{\text {a }} C E$ clinically evaluable, ICU intensive care unit, $L F U$ late follow-up, MITT modified

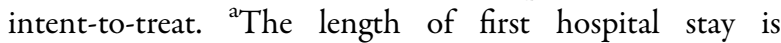
calculated as number of days from randomization until when the patient is no longer recorded as being in one of the hospital wards (including ICU) for the first admission. The length of ICU stay is calculated as number of days from randomization until when the patient is no longer recorded as being in ICU for the first admission. For patients who stay in hospital (including ICU) beyond the LFU visit, the length of stay is censored at the LFU visit. For patients lost to follow-up while in a hospital ward (including ICU), the length of stay is censored at the last recorded visit
[18]. A greater proportion of ICU patients also had bacteremia. However, the incidence of SIRS in the ICU subset was lower than in non-ICU patients, and a greater proportion of non-ICU patients received prior systemic antibiotics within the 4 weeks prior to administration of study drug. Similar proportions of patients in the ICU and non-ICU subsets had comorbid conditions ( $\sim 26 \%$ vs. $\sim 24 \%$, respectively). These results are in contrast with other studies, which have demonstrated an increased rate of ICU admissions among patients with comorbid conditions. For example, a nationwide population-based study in Taiwan identified that multiple comorbid conditions were associated

Table 4 Cox proportional hazards model for analysis of length of first hospital stay (MITT and CE populations)

\begin{tabular}{|c|c|c|c|c|c|}
\hline \multicolumn{2}{|l|}{ Exploratory variables } & \multicolumn{2}{|c|}{ MITT population } & \multicolumn{2}{|l|}{ CE population } \\
\hline Reference category & Categories tested & $\begin{array}{l}\text { Hazard ratio } \\
(95 \% \mathrm{CI})\end{array}$ & $P$ value & $\begin{array}{l}\text { Hazard ratio } \\
(95 \% \mathrm{CI})\end{array}$ & $P$ value \\
\hline
\end{tabular}

Treatment

Vancomycin + aztreonam $\quad$ Ceftaroline fosamil $\quad 1.074(0.919,1.257) \quad 0.368 \quad 1.098(0.924,1.305) \quad 0.288$

Age group

$<65$ years $\quad \geq 65$ years $\quad 1.042(0.875,1.240) \quad 0.647 \quad 0.968(0.797,1.176) \quad 0.746$

Gender

Female

Male

$0.958(0.823,1.115)$

0.581

$0.941(0.795,1.113)$

0.478

Geographical region

European Union

North America
Other European
Latin America
Asia

Rest of the World

$\begin{array}{lrrr}2.135(1.626,2.803) & <0.001 & 2.870(2.055,4.009) & <0.001 \\ 1.137(0.906,1.429) & 0.268 & 1.120(0.877,1.430) & 0.365 \\ 1.161(0.883,1.527) & 0.285 & 1.141(0.848,1.535) & 0.384 \\ 0.630(0.503,0.790) & <0.001 & 0.681(0.534,0.869) & 0.002 \\ 1.856(1.324,2.603) & <0.001 & 1.776(1.177,2.681) & 0.006\end{array}$

Presence of comorbidities

No

Yes

$0.736(0.621,0.873)$

$<0.001$

$0.752(0.621,0.910)$

0.003

Hospital location

Hospital ward

ICU

Emergency room
$0.389(0.269,0.562)$

$<0.001$

$0.376(0.250,0.566)$

$<0.001$

$0.984(0.716,1.352)$

$0.921 \quad 0.721(0.473,1.099)$

0.128

Cox proportional hazards model includes explanatory variables: treatment (ceftaroline fosamil or vancomycin plus aztreonam), age, group, gender, geographic region, presence of comorbidities, and hospital location $C E$ clinically evaluable, $C I$ confidence interval, $M I T T$ modified intent-to-treat, TOC test-of-cure 
with an increased rate of ICU admission in patients hospitalized with cSSTI [19]. Among comorbid conditions in patients assessed in the Taiwan study, diabetes mellitus was the most commonly identified [19].

In line with the overall COVERS population [13] and the non-ICU subset, the most frequently isolated pathogens from patients in the ICU subset included $S$. aureus, streptococci, and Enterobacteriaceae. These findings are consistent with other prospective clinical studies in patients with cSSTI or ABSSSI $[11,12,20]$ and retrospective epidemiological data [7, 21]. Ceftaroline fosamil and vancomycin plus aztreonam were highly active in vitro against the most common pathogens isolated from patients in the ICU subset. In the overall population, ceftaroline fosamil and vancomycin plus aztreonam also demonstrated in vitro activity against the most commonly isolated baseline pathogens, including both MSSA and MRSA [13].

Unlike the overall COVERS study, and the much larger non-ICU subset, there were some imbalances in some patient and disease characteristics within the ICU subset which should be considered when interpreting the clinical outcomes. For example, in the vancomycin plus aztreonam group, patients were older, proportionally more patients presented with bacteremia at baseline, and median lesion size was almost double that in the ceftaoline fosamil group; a higher proportion of patients in the ceftaroline fosamil group received prior systemic antibiotics within the 4 weeks before first dose of study therapy. These differences, combined with the small sample size of the ICU subset, mean interpreting the results for median duration of antibiotic treatment and ICU LOS, which were shorter for ceftaroline fosamil than for vancomycin plus aztreonam (in contrast to median treatment duration and overall hospital LOS in non-ICU patients, which were similar between groups), is challenging. Nonetheless, clinical cure rates at TOC were generally favorable for ICU and non-ICU patients treated with ceftaroline fosamil or vancomycin plus aztreonam, and the frequency and severity of adverse events were also generally consistent between treatment groups and between ICU and non-ICU patients. As might be expected given the indicators of more severe disease in ICU patients, in the ceftaroline fosamil group, clinical cure rates at TOC were higher for nonICU patients than for those in the ICU subset. In contrast, for vancomycin plus aztreonam, the opposite trend was observed, with higher cure rates in the ICU subset relative to non-ICU patients. These unexpected findings may reflect the baseline and disease imbalances between treatment groups in the ICU subset noted above; they also highlight the limitations of this analysis in terms of the small sample size of the ICU subset, which comprised $<6 \%$ of the overall COVERS population.

There was no difference between treatment groups in the median hospital LOS (11 days in both the MITT and CE populations) in this analysis. However, ICU admission was a risk factor for increased hospital LOS. These findings are consistent with those of a retrospective observational study, in which patients with necrotizing fasciitis, abscess, or cellulitis had median ICU LOS of 8, 14, and 21 days, respectively [22]. In the REACH study, median hospital LOS for patients admitted to the ICU was 25 days versus 11 days for patients not admitted to the ICU [7].

\section{CONCLUSIONS}

In conclusion, patients with cSSTI admitted to the ICU in the phase 3 COVERS trial had baseline and disease characteristics reflective of more severe infection compared with non-ICU patients: more patients had bacteremia and burn infections, and median lesion size was larger. There were also notable differences between treatment groups within the ICU subset. Both ceftaroline fosamil and vancomycin plus aztreonam achieved favorable treatment outcomes in these more severely ill ICU patients, with a shorter ICU LOS for ceftaroline fosamil, although the small numbers of patients and baseline imbalances between groups limit the generalizability of the data. Further studies in the cSSTI ICU patient population are warranted to allow definitive conclusions. 


\section{ACKNOWLEDGEMENTS}

Funding. The COVERS study was originally sponsored by AstraZeneca and is now sponsored by Pfizer. AstraZeneca's rights to ceftaroline fosamil were acquired by Pfizer in December 2016. Funding for the journal's Rapid Service Fees was provided by Pfizer.

Medical Writing/Editorial Assistance. Medical writing support was provided by Mark Waterlow BSc, CMPP of Prime, Knutsford, Cheshire, UK, funded by Pfizer.

Authorship. All named authors meet the International Committee of Medical Journal Editors (ICMJE) criteria for authorship for this article, take responsibility for the integrity of the work as a whole, and have given their approval for this version to be published.

Authorship Contributions. Matthew Dryden was principal investigator for the COVERS study. Jean Li Yan coordinated the programming and statistical analyses. All authors were involved in data interpretation and drafting the manuscript for important intellectual content.

Prior Presentation. An abstract and poster summarising the data in this manuscript were presented at the 29th European Congress of Clinical Microbiology and Infectious Diseases, 13-16 April 2019, Amsterdam, The Netherlands.

Disclosures. Miguel Sánchez-García has received speaker's fees from Pfizer. Matthew Dryden received institutional research funding from AstraZeneca for the conduct of the study. Jennifer Hammond, Jean Li Yan, Wajeeha Ansari, and Michal Kantecki are employees of Pfizer and hold Pfizer shares/and or stock options.

Compliance with Ethics Guidelines. The protocol was approved by each study site's independent ethics committees/institutional review board, and the study was conducted in accordance with the Declaration of Helsinki and the International Conference on
Harmonization/Good Clinical Practice. All patients provided written informed consent.

Data Availability. Upon request, and subject to certain criteria, conditions and exceptions see (https://www.pfizer.com/science/ clinical-trials/trial-data-and-results for more information), Pfizer will provide access to individual de-identified participant data from Pfizersponsored global interventional clinical studies conducted for medicines, vaccines and medical devices (1) for indications that have been approved in the US and/or EU or (2) in programs that have been terminated (i.e., development for all indications has been discontinued). Pfizer will also consider requests for the protocol, data dictionary, and statistical analysis plan. Data may be requested from Pfizer trials 24 months after study completion. The deidentified participant data will be made available to researchers whose proposals meet the research criteria and other conditions, and for which an exception does not apply, via a secure portal. To gain access, data requestors must enter into a data access agreement with Pfizer.

Open Access. This article is licensed under a Creative Commons Attribution-NonCommercial 4.0 International License, which permits any non-commercial use, sharing, adaptation, distribution and reproduction in any medium or format, as long as you give appropriate credit to the original author(s) and the source, provide a link to the Creative Commons licence, and indicate if changes were made. The images or other third party material in this article are included in the article's Creative Commons licence, unless indicated otherwise in a credit line to the material. If material is not included in the article's Creative Commons licence and your intended use is not permitted by statutory regulation or exceeds the permitted use, you will need to obtain permission directly from the copyright holder. To view a copy of this licence, visit http://creativecommons.org/licenses/by$\mathrm{nc} / 4.0 /$. 


\section{REFERENCES}

1. Stevens DL, Bisno AL, Chambers HF, et al. Practice guidelines for the diagnosis and management of skin and soft tissue infections: 2014 update by the Infectious Diseases Society of America. Clin Infect Dis. 2014;59(2):e10-e52.

2. Eckmann C, Dryden M. Treatment of complicated skin and soft-tissue infections caused by resistant bacteria: value of linezolid, tigecycline, daptomycin and vancomycin. Eur J Med Res. 2010;15(12): 554-63.

3. DiNubile MJ, Lipsky BA. Complicated infections of skin and skin structures: when the infection is more than skin deep. J Antimicrob Chemother. 2004;53(Suppl 2):ii37-50.

4. Vincent J-L, Rello J, Marshall J, et al. International study of the prevalence and outcomes of infection in intensive care units. JAMA. 2009;302(21): 2323-9.

5. Burnham JP, Kirby JP, Kollef MH. Diagnosis and management of skin and soft tissue infections in the intensive care unit: a review. Intensive Care Med. 2016;42(12):1899-911.

6. Cranendonk DR, van Vught LA, Wiewel MA, et al. Clinical characteristics and outcomes of patients with cellulitis requiring intensive care. JAMA Dermatol. 2017;153(6):578-82.

7. Garau J, Ostermann H, Medina J, et al. Current management of patients hospitalized with complicated skin and soft tissue infections across Europe (2010-2011): assessment of clinical practice patterns and real-life effectiveness of antibiotics from the REACH study. Clin Microbiol Infect. 2013;19(9):E377-E385385.

8. Nichols RL, Florman S. Clinical presentations of soft-tissue infections and surgical site infections. Clin Infect Dis. 2001;33(Suppl 2):S84-S93.

9. Garrison MW, Kawamura NM, Wen MM. Ceftaroline fosamil: a new cephalosporin active against resistant Gram-positive organisms including MRSA. Expert Rev Anti Infect Ther. 2012;10(10):1087-103.

10. Scott LJ. Ceftaroline fosamil: a review in complicated skin and soft tissue infections and community-acquired pneumonia. Drugs. 2016;76(17): 1659-74.

11. Corey GR, Wilcox MH, Talbot GH, Thye D, Friedland D, Baculik T. CANVAS 1: the first Phase III, randomized, double-blind study evaluating ceftaroline fosamil for the treatment of patients with complicated skin and skin structure infections.
J Antimicrob Chemother. 2010;65(Suppl 4): iv41-iv51.

12. Wilcox MH, Corey GR, Talbot GH, Thye D, Friedland D, Baculik T. CANVAS 2: the second phase III, randomized, double-blind study evaluating ceftaroline fosamil for the treatment of patients with complicated skin and skin structure infections. J Antimicrob Chemother. 2010;65(suppl 4): iv53-iv65.

13. Dryden M, Zhang Y, Wilson D, Iaconis JP, Gonzalez J. A Phase III, randomized, controlled, non-inferiority trial of ceftaroline fosamil $600 \mathrm{mg}$ every $8 \mathrm{~h}$ versus vancomycin plus aztreonam in patients with complicated skin and soft tissue infection with systemic inflammatory response or underlying comorbidities. J Antimicrob Chemother. 2016;71(12):3575-84.

14. Cockcroft DW, Gault MH. Prediction of creatinine clearance from serum creatinine. Nephron. 1976;16(1):31-41.

15. Food and Drug Administration. Guidance for Industry Acute Bacterial Skin and Skin Structure Infections: Developing Drugs for Treatment. 2013. https://www.fda.gov/downloads/Drugs/Guidances/ ucm071185.pdf. Accessed 22 Nov 2018.

16. European Committee on Antimicrobial Susceptibility Testing (EUCAST). Clinical breakpoints for bacteria, v 9.0. 2019. https://www.eucast.org/ clinical_breakpoints/. Accessed 11 Nov 2019.

17. Clinical and Laboratory Standards Institute (CLSI). Performance standards for antimicrobial susceptibility testing. Twenty-ninth informational supplement M100-S29. 2019. https://clsi.org/standards/ products/microbiology/documents/m100/. Accessed 21 Jun 2019.

18. Ki V, Rotstein C. Bacterial skin and soft tissue infections in adults: A review of their epidemiology, pathogenesis, diagnosis, treatment and site of care. Can J Infect Dis Med Microbiol. 2008;19(2):173-84.

19. Shen HN, Lu CL. Skin and soft tissue infections in hospitalized and critically ill patients: a nationwide population-based study. BMC Infect Dis. 2010;10: 151.

20. Claeys KC, Zasowski EJ, Trinh TD, et al. Open-Label Randomized trial of early clinical outcomes of Ceftaroline Fosamil versus vancomycin for the treatment of acute bacterial skin and skin structure infections at risk of methicillin-resistant Staphylococcus aureus. Infect Dis Ther. 2019;8(2):199-208.

21. Zervos MJ, Freeman K, Vo L, et al. Epidemiology and outcomes of complicated skin and soft tissue 
infections in hospitalized patients. J Clin Microbiol. 2012;50(2):238-45.

22. Malheiro LF, Magano R, Ferreira A, Sarmento A, Santos L. Skin and soft tissue infections in the intensive care unit: a retrospective study in a tertiary care center. Rev Bras Ter Intensiva. 2017;29(2): 195-205. 